PALEO

Revue d'archéologie préhistorique

Numéro spécial | 2009-2010

Entre le marteau et l'enclume...

\title{
La percussion directe dure au campaniforme : modalités d'application et difficultés d'interprétation
}

Hard direct percussion of the Bell Beaker culture: variabilities of its application and difficulties in its interpretation

\section{Robin Furestier}

\section{OpenEdition}

Journals

Édition électronique

URL : http://journals.openedition.org/paleo/1986

DOI : $10.4000 /$ paleo.1986

ISSN : 2101-0420

Éditeur

SAMRA

Édition imprimée

Pagination : 189-200

ISSN : 1145-3370

Référence électronique

Robin Furestier, «La percussion directe dure au campaniforme : modalités d'application et difficultés d'interprétation », PALEO [En ligne], Numéro spécial | 2009-2010, mis en ligne le 12 avril 2012, consulté le 07 juillet 2020. URL : http://journals.openedition.org/paleo/1986 ; DOI : https://doi.org/10.4000/ paleo.1986

Ce document a été généré automatiquement le 7 juillet 2020.

\section{c) (1) $(9)$}

PALEO est mis à disposition selon les termes de la licence Creative Commons Attribution - Pas d'Utilisation Commerciale - Pas de Modification 4.0 International. 


\title{
La percussion directe dure au campaniforme : modalités d'application et difficultés d'interprétation
}

\author{
Hard direct percussion of the Bell Beaker culture: variabilities of its application \\ and difficulties in its interpretation
}

Robin Furestier

1 En tant que geste d'une grande simplicité, accessible des premiers hominidés à nos propres contemporains, la percussion directe à la pierre dure cumule elle-même l'ensemble des qualificatifs dépréciatifs des spécialistes et la vision péjorative du quotidien préhistorique $\mathrm{du}$ grand public. La Préhistoire récente, et plus particulièrement le Campaniforme, ne déroge pas à ce constat : la présence de l'élément céramique et son perfectionnement technique et esthétique n'ont fait qu'accentuer le contraste entre deux productions pourtant complémentaires et culturellement riches de sens.

2 Toutefois, envisager l'approche technologique de la percussion directe dure n'est pas uniquement un moyen pour remettre cet aspect technique au cœur des débats, il contribue avant tout au développement d'un outil d'analyse peu utilisé jusqu'à présent pour appréhender l'évolution des comportements socio-économiques des groupes humains de la fin du troisième millénaire avant notre ère et la diffusion des traditions techniques et culturelles campaniformes.

3 L'exemple du Sud-Est de la France est apparu potentiellement apte à répondre aux besoins d'une étude technologique de l'industrie lithique de cette période. À la croisée d'importants chemins culturels (Espagne, Europe centrale et Italie) et physiques (mondes alpins, marins et fluviaux), le Sud-Est de la France constitue une région présentant une expression régionale du Campaniforme (le Rhodano-provençal) et aussi un recensement récent de plus de 300 sites (Lemercier 2004) qui témoignent de sa 
grande variabilité archéologique (sites perchés, de plaine, de grotte... d'habitat ou sépulcraux) et chronoculturelle (campaniforme ancien, régional et épicampaniforme). L'ensemble de ces facteurs a permis de réaliser une étude représentative des caractéristiques lithiques de cette région, de proposer une évolution de cette dernière, et d'en faire un élément de comparaison avec le reste de l'Europe campaniforme (Furestier 2005 et 2007).

Cet exercice a été mené d'un point de vue technologique global, et non plus selon une approche exclusivement typologique. Il a donc été possible de développer l'axe de recherche consacré aux techniques de taille mises en œuvre par les Campaniformes, négligées jusqu'alors. En effet, d'un point de vue historiographique, le Néolithique - et particulièrement sa fin - n'a pas constitué la période la plus investie par les études portant sur la percussion directe à la pierre dure. Les productions spécialisées et les pièces d'exception diffusées sur de grandes distances pendant le troisième millénaire (grandes lames et poignards en silex oligocène ou turonien essentiellement) ont occulté pendant longtemps les productions plus simples souvent réunies sous le terme très discuté d' « expédientes » (Astruc 2005 ; Astruc et al. 2006).

5 L'aspect polymorphe de la percussion directe à la pierre dure constitue une limite à la mise en évidence de récurrences et de spécificités fortes. Cela explique en partie le désintérêt pour cette technique à cette période de la Préhistoire. Reconnaître la technique de la percussion directe à la pierre dure par la présence de quelques percuteurs, d'éclats et de nucléus informes est longtemps resté la règle dans les études concernant les séries du Néolithique final. Or, la présente table ronde a montré que les modalités d'application de cette technique de taille sont diverses et peuvent constituer des caractéristiques culturelles spécifiques.

\section{La percussion directe à la pierre dure}

Malgré sa généralisation à l'ensemble des périodes de la Préhistoire, les critères de reconnaissance de la percussion directe dure ne sont précisément définis que depuis peu (Pelegrin 2000). La nature ubiquiste de cette technique semble interdire toute attribution chronoculturelle. Si des tendances peuvent être proposées entre le Paléolithique et le Néolithique, notamment en ce qui concerne le module des éclats produits, il est difficile de différencier les variabilités de mise en œuvre de la percussion directe dure au sein du Néolithique, et particulièrement au Néolithique final. C'est ici encore une limite expliquant le peu d'intérêt que suscite cette technique au sein des recherches sur la fin du troisième millénaire.

7 Toutefois, l'étude de l'industrie lithique campaniforme a permis de mettre en évidence au moins deux percussions directes dures différentes et des modes d'application spécifiques pour la deuxième.

8 La première percussion est bien connue et sa mise en œuvre est responsable de la majorité des supports produits observés dans les séries lithiques du Sud-Est de la France. Il s'agit d'une percussion directe dure lancée, dirigée majoritairement vers la production de petits éclats d'un module compris entre 15 et $35 \mathrm{~mm}$ (fig. 1), et - dans une moindre mesure - vers la mise en forme de préformes d'armatures, le façonnage de quelques pièces à retouches marginales, voire de quelques autres outils. Mais la principale mise en œuvre de la percussion directe dure reste la production de petits 
éclats (fig. 2) à partir de supports de base pouvant être des fragments de blocs, des galets ou des éclats débités sur les gîtes de matières premières.

Figure 1 - Diagramme de rapport longueur/largeur des éclats entiers (en $\mathrm{mm}$ ) du site du Mas de Vignole IV à Nîmes, Gard

Figure 1 - Length/weigth connection diagram ( $\mathrm{mm}$ ) of flakes to the Mas de Vignole IV, Nîmes-Gard site

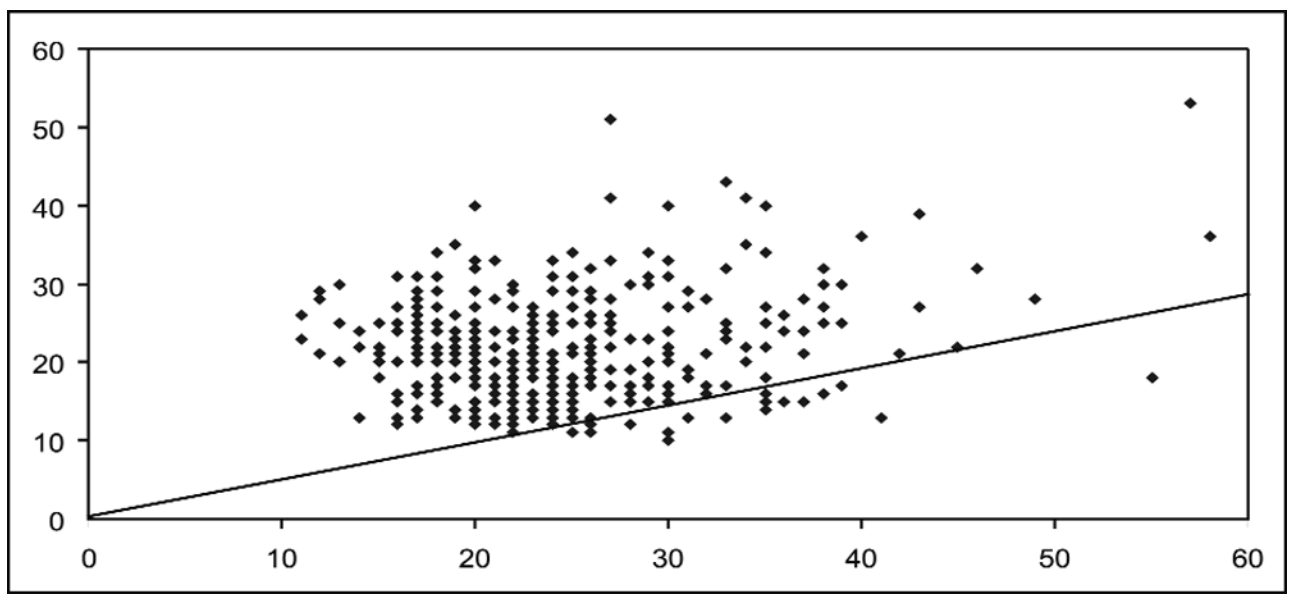

Figure 2 - Nucléus et éclats campaniformes débités par percussion directe dure. [Le Mas de Vignole IV à Nîmes, Gard (photo R. Furestier)] Figure 2 - Bell beaker cores and flakes produced by hard direct percussion

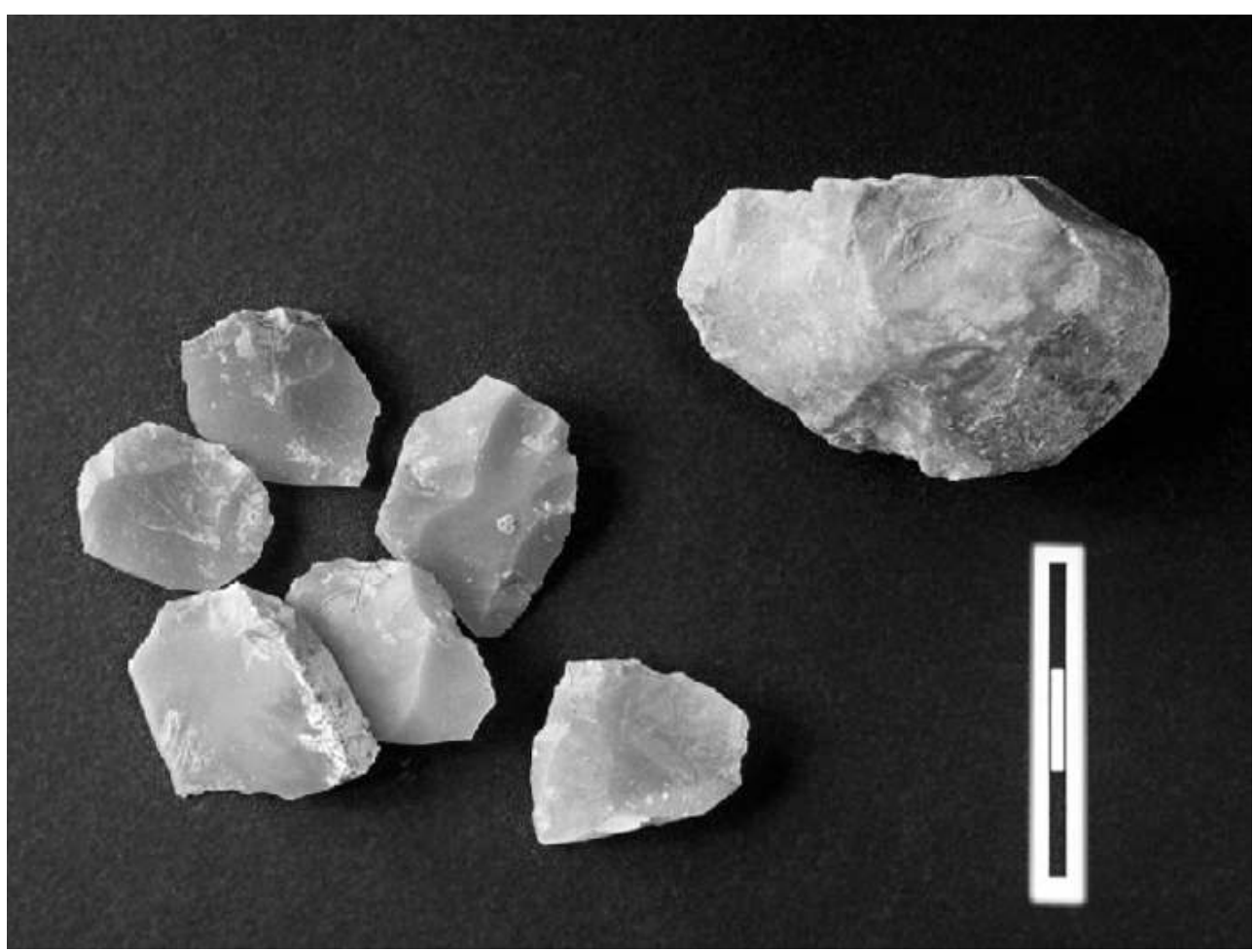

9 Cet approvisionnement “au plus près" privilégie le silex mais n'interdit pas la diversité d'acquisition de roches taillables situées dans le même territoire exploité. Cette variabilité dans l'approvisionnement en matières premières lithiques peut également être envisagée comme une logique d'optimisation des déplacements ayant pour but l'acquisition de matières premières de natures variées. Les principales ressources acquises (silex, argiles, vestiges fauniques) proviennent en effet de la sphère 
locale (Convertini 1996; Convertini et al. 2004), ce qui peut encourager l'hypothèse de l'exploitation du territoire sous forme de collectes cumulées (Perlès 1991). La logique dominante d'exploitation des ressources siliceuses locales a souvent des répercussions sur la qualité des matières premières mises en œuvre. La taillabilité plus que l'excellence de la matière semble recherchée. Les choix des supports de nucléus ne constituent donc pas un critère discriminant. Seul un module du support de base semble recherché (généralement inférieur à $10 \mathrm{~cm}$ ). Ainsi, des galets dépassant le module recherché ont pu être fragmentés afin d'utiliser les éclats produits comme supports de nucléus. L'approvisionnement en matières premières représente donc un réel choix, confirmé par le fait que des silex de bonne qualité et de gros module étaient disponibles mais n'ont pas été prélevés.

10 En ce qui concerne les dimensions des nucléus, établir une moyenne globale et commune ne constitue pas une information archéologiquement significative mais une tendance indicative peut être proposée. Ainsi, une classe moyenne comprise entre 35 et $60 \mathrm{~mm}$ peut regrouper la majorité des nucléus de chaque série (fig. 3). Néanmoins, les nucléus présentant des dimensions inférieures à cette moyenne apparaissent également comme une caractéristique commune des industries lithiques campaniformes. Cette réduction des dimensions peut atteindre des extrêmes avec des nucléus très petits (fig. $3 \mathrm{n}^{\circ} 2$ ) présentant des négatifs d'enlèvements quelquefois inférieurs au centimètre, ce qui génère des problèmes d'interprétation quant à la nature même des pièces concernées. Typologiquement, ces pièces ne peuvent être classées dans une catégorie d'outil même si le risque de confusion reste possible notamment entre grattoirs et nucléus (Gassin et al. 2004). Les enlèvements, même peu nombreux, ne peuvent être interprétés comme des retouches. Ils témoignent d'une percussion directe dure portée à l'intérieur d'un plan de frappe distinct. En considérant son adaptation à ce type de percussion, la découverte d'un petit percuteur en silex (fig. $\left.3 n^{\circ} 7\right)$, plus adapté à ces modules, peut également confirmer l'intentionnalité de ce type de débitage. Si l'intention semble réelle, le but de production reste néanmoins problématique. L'observation des négatifs d'enlèvements des «produits » issus de ces nucléus révèle des caractéristiques difficilement assimilables à la définition de support. Aucun outil typologique ne peut d'ailleurs attester l'utilisation de ces éclats. De plus, hormis cette difficulté interprétative, les dimensions de ces pièces posent des problèmes d'ordre technique : la percussion et surtout le maintien de ces pièces sont effectivement complexes.

11 La majorité des nucléus sont débités par percussion lancée «classique » (fig. 3). Sous cette appellation générique, on regroupe les percussions directes lancées sur nucléus tenus dans la main ou calés sur la cuisse. Le débitage est organisé à partir d'un plan de frappe unique ou préférentiel qui est généralement lisse et constitué d'une face naturelle, d'une face inférieure, ou aménagé par l'enlèvement d'un éclat. Plusieurs séries témoignent d'ailleurs de la présence de nucléus dont le plan de frappe est dégagé par un enlèvement rebroussant. À partir de ces plans, le débitage se développe sur un ou plusieurs fronts, sans organisation visible entre eux. L'exploitation des nucléus par percussion directe dure génère des morphologies multiples mais les tendances pyramidales, pseudo-pyramidales, voire polyédriques, sont les plus fréquemment observées.

12 Au regard de ces cas, il est possible de proposer une évolution de ces morphologies en terme de schéma opératoire: après l'exploitation du nucléus à partir d'un plan de 
frappe unique, un autre plan offrant un angle favorable au détachement d'éclats est utilisé, puis suivant la même logique, tous les angles favorables au détachement d'un éclat sont exploités. La morphologie polyédrique qui en résulte caractériserait alors l'exploitation optimale des nucléus (Detrey 2002) et serait liée à un état d'exhaustion. Des nuances doivent toutefois être apportées à cette proposition. Ce schéma n'est pas systématique et des productions développées exclusivement à partir d'un plan de frappe unique sont fréquentes et confirmées par l'étude des faces supérieures des éclats. On remarque également l'exploitation modérée - voire faible - du potentiel de nombreux nucléus qui montrent des séquences courtes même dans des cas où la qualité de la matière première permet la poursuite du débitage. Le caractère principal du schéma opératoire campaniforme semble donc résider dans un réel opportunisme de production, sans toutefois que toutes les étapes de ce schéma puissent encore être expliquées.

Figure 3 - Nucléus campaniformes débités par percussion directe dure lancée et percuteur en silex [1 et 4 - Le Mas de Vignole IV à Nîmes, Gard ; 2 et 7 : Le Fortin-du-Saut à Châteauneuf-lèsMartigues, Bouches-du-Rhône ; 3 : Les Calades 2 à Eyguières, Bouches-du-Rhône ; 5 : L'abri du Capitaine à Sainte-Croix du Verdon, Alpes-de-Haute-Provence ; 6 : La Grotte Murée à Montpezat, Alpes-de-Haute-Provence (dessins R. Furestier)]

Figure 3 - Bell beaker cores exploited by hard direct percussion and flint hammer.

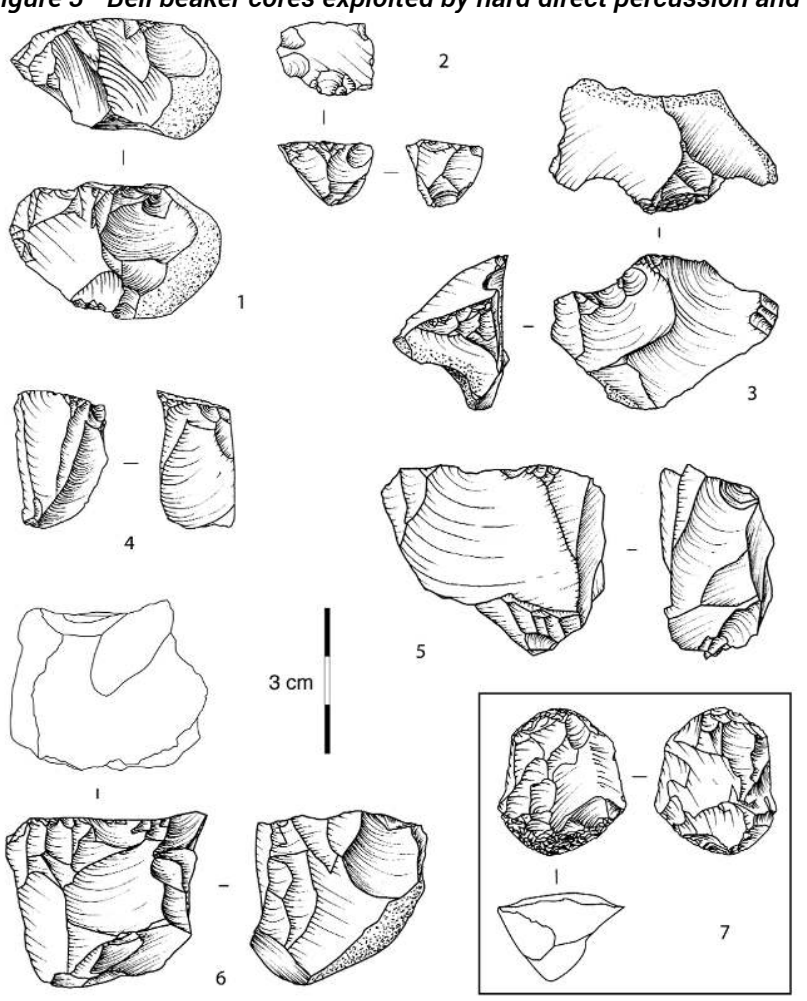

Enfin, on ne peut pas exclure la possibilité d'une recherche de la morphologie polyédrique qui traduirait alors un changement de statut de ces nucléus. La fin de leur exploitation pourrait être corrélée avec un objectif typologique: la réalisation de polyèdres (fig. $4 \mathrm{n}^{\circ}$ 2) même si cette finalité est observée seulement en contexte néolithique ancien jusqu'à présent (Binder 1987 ; Allard 2005).

Quel que soit son objectif final, la percussion directe dure représente la technique dominante des tailleurs campaniformes. En revanche, une certaine diversité des 
modalités d'application de cette technique est observée et caractérisée par la percussion sur enclume.

Figure 4 - Nucléus campaniformes débités par percussion directe dure sur enclume.

[1 : Le Fortin-du-Saut à Châteauneuf-lès-Martigues, Bouches-du-Rhône ; 2 : Le Mas de Vignole IV à Nîmes, Gard ; 3 : Les Calades 1 à Eyguières, Bouches-du-Rhône ; 4 : Georges Besse II à Nîmes, Gard (dessins R. Furestier)]

Figure 4 - Bell beaker cores and flakes exploited by hard direct percussion on anvil

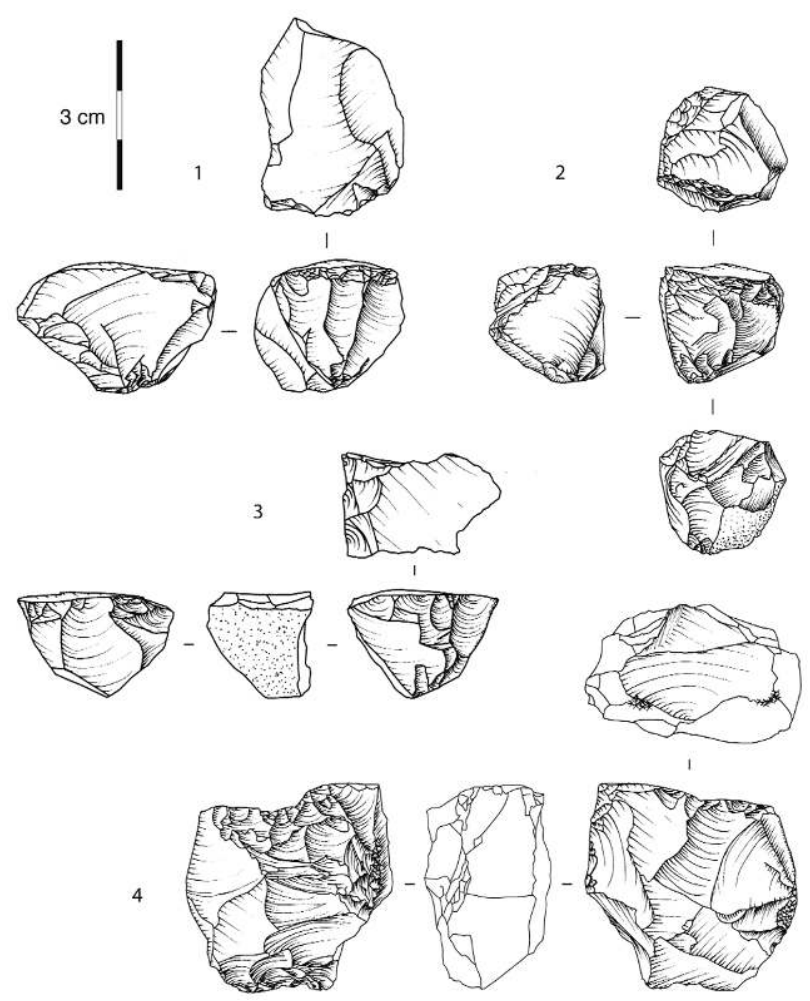

\section{La percussion sur enclume}

Outre ses mises en œuvre durant le Paléolithique (voir les nombreux articles de ce volume), le débitage sur enclume est effectivement présent sur l'ensemble du Néolithique (Guyodo et Marchand 2005) et jusqu'à l'âge du Bronze (Binder 1987 ; Lech 1982/1983). Reconnaître en marge de la percussion directe dure lancée «classique », une percussion sur enclume dans des séries lithiques de contexte campaniforme ne constitue donc pas, en soi, une nouveauté. Elle est notamment définie pour la zone atlantique comme un "débitage côtier " (Joussaume 1981) et assez souvent observée (Guyodo et Marchand 2005). L'aspect ubiquiste de ce type de débitage est alors interprété par Joussaume comme une technique adaptée à la nature de la matière première, comme un moyen de tester les galets de mauvaise qualité ou encore, comme un moyen mis en œuvre par le tailleur pour ne pas se taper sur les doigts (Joussaume 1981). Ces explications uniquement techniques sont avancées par l'auteur pour marquer l'absence de choix culturel.

Néanmoins, le cas du Sud-Est de la France permet d'enrichir la caractérisation de cette technique pour la fin du troisième millénaire et de la faire apparaître comme un élément signifiant des caractéristiques socio-culturelles des Campaniformes (Furestier 
2005 ; Guyodo et Marchand 2005). D'une présence plus ou moins marquée, la mise en œuvre de cette technique est constatée dans presque toutes les séries étudiées. La nouveauté réside alors moins dans son observation que dans la distinction de deux modes d'application de cette technique. Ils sont ici définis simplement par type et résumés schématiquement :

- Type 1 : la percussion intérieure oblique,

- Type 2 : la percussion verticale d'éclatement ${ }^{1}$

Selon le type 1, la percussion (fig. 6) est appliquée lancée sur un plan de frappe lisse d'un nucléus globuleux, polyédrique ou pyramidal maintenu sur l'enclume. La percussion est rentrante et détache des éclats à talons lisses et larges. Le désaxement plus ou moins prononcé entre le point de percussion et le point d'ancrage sur l'enclume génère un contrecoup peu marqué, voire invisible pour les premiers éclats qui n'emportent pas la totalité du front de débitage. La distinction entre ces premiers produits et le reste des éclats obtenus par percussion directe dure lancée "classique » est alors difficile. Les nucléus présentent, en revanche, des écrasements distaux visibles.

Figure 6 - Schématisation de la percussion intérieure oblique sur enclume (type 1) Figure 6 - Scheme of oblique interior percussion on anvil (type 1)

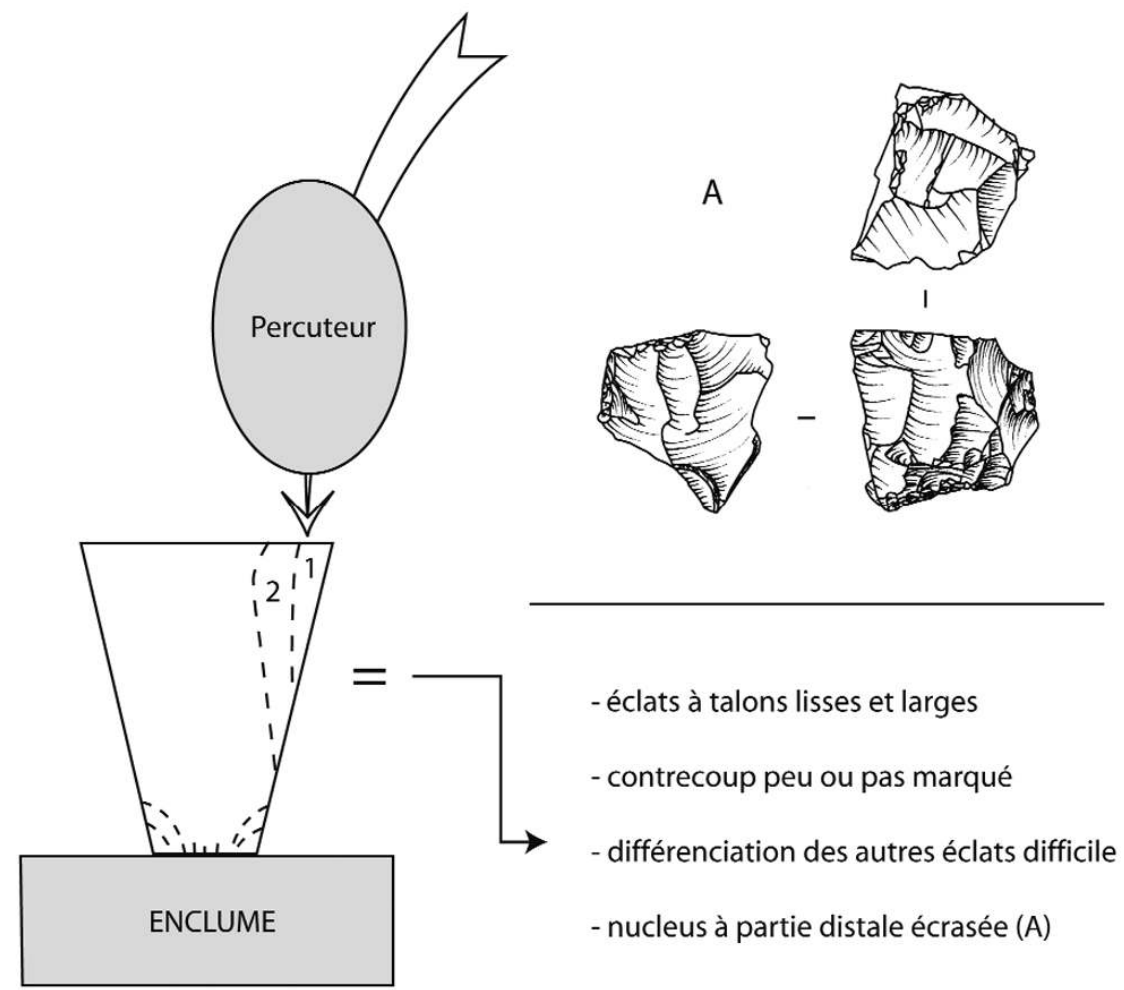

Selon le type 2, la percussion (fig. 7) est appliquée lancée sur un plan de frappe de surface plus restreinte, voire sur une arête et dans un axe vertical opposé au point d'appui sur l'enclume. Le contrecoup est alors maximum. 
Figure 7 - Schématisation de la percussion verticale d'éclatement sur enclume (type 2) Figure 7 - Scheme of straight down splintering percussion on anvil (type 2)

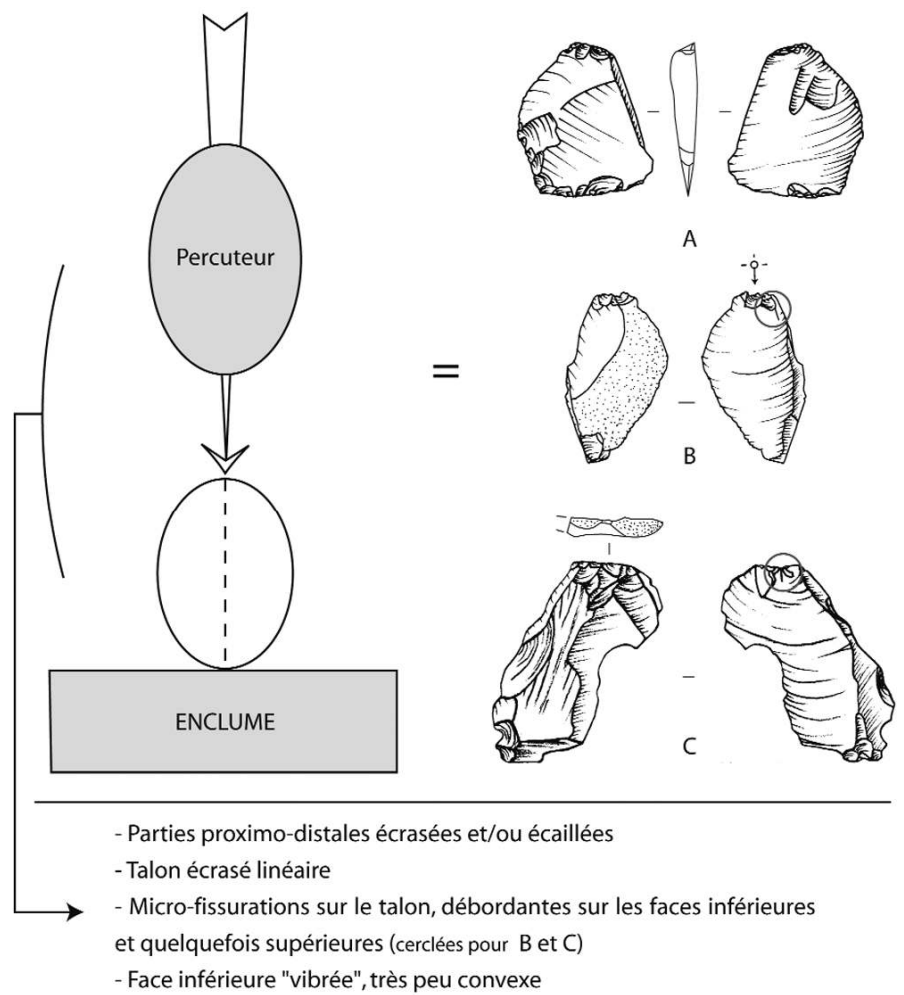

Ces deux modes d'application sont attestés par des nucléus de deux types (fig. 4):

- pour la percussion intérieure oblique, des nucléus pyramidaux ou polyédriques qui présentent des stigmates au point d'appui sur l'enclume: surface restreinte irrégulière et écrasée à micro-fissurations rapprochées ;

- pour la percussion verticale d'éclatement, des nucléus longitudinaux ou polyédriques présentant deux surfaces opposées et écrasées et un ou plusieurs fronts de débitage également opposés.

L'examen de ces deux exemples de nucléus illustre bien l'importante variabilité des modes opératoires de la percussion sur enclume. De plus, on ne peut pas exclure la possibilité de reprise d'un nucléus par percussion de type 2, initialement débité par percussion de type 1 .

21 Ces deux techniques peuvent créer divers types de supports, plus ou moins caractérisables. Certains éclats apparaissent alors comme des moyens complémentaires de reconnaissance de la percussion sur enclume.

Nombre d'éclats produits par percussion de type 1 sont peu différenciables de ceux produits par percussion directe dure «classique». La faiblesse du contrecoup ne contrarie pas la diffusion de l'onde de fracture dans la matière et les éclats qui ne se développent pas jusqu'à l'extrémité distale du nucléus ne peuvent être isolés du cortège des éclats débités par percussion directe dure.

En revanche, les éclats se développant sur toute la longueur du nucléus ou les éclats issus d'un débitage par percussion de type 2 présentent plus de spécificités. La verticalité du coup, et surtout l'opposition plan de frappe/point d'appui, assurent un effet de contrecoup très marqué. Les éclats produits présentent de nombreux stigmates 
caractéristiques (fig. 5 et 7), même s'ils n'emportent qu'une partie du front de débitage ${ }^{2}$ :

- leurs parties proximo-distales sont écrasées. Les talons ont donc une morphologie écrasée linéaire irrégulière et peuvent porter des fissurations pénétrantes de quelques millimètres maximum (fig. $6 \mathrm{~B}$ et $\mathrm{C}$ ) témoignant de la violence et de la répétition des coups portés et/ou de l'élargissement de la surface de contact nucléus/percuteur. Ces fissurations sont visibles sur la face inférieure et quelquefois sur la face supérieure. L’aspect écrasé est souvent plus discret sur la partie distale qui peut présenter quelques écaillements opposés à l'axe de débitage de l'éclat. Selon la morphologie du nucléus, certains éclats courts peuvent présenter une partie distale exempte de tout écrasement.

- leurs faces inférieures sont parcourues d'ondes très prononcées dites «vibrées» (Binder 1987) dues au contrecoup et sont souvent d'une convexité peu marquée, sans bulbe apparent, quelquefois plates voire concaves (fig. $5 \mathrm{n}^{\circ} 5$ par exemple).

- enfin, les faces supérieures offrent également quelques informations. Elles peuvent porter des négatifs de directions opposées (proximo-distaux/disto-proximaux) témoignant d'enlèvements d'éclats axiaux de première intention et du détachement d'éclats d'axe opposé dus au contrecoup (fig. $5 \mathrm{n}^{\circ}$ ). Toutefois, cette caractéristique doit être relativisée par la possibilité d'interprétation de retournement du nucléus sur l'enclume générant un réel débitage bipolaire.

En marge de ces caractéristiques générales, on peut ajouter une remarque sur le nombre d'éclats rebroussés issus du débitage par percussion sur enclume. Les difficultés liées au maintien du nucléus et les contraintes intrinsèques de cette technique peuvent expliquer la verticalité de la percussion et donc, la fréquence des éclats rebroussés dont l'angle de chasse approche souvent les $90^{\circ}$.

Selon les critères de reconnaissance définis ici, la percussion sur enclume n'est que partiellement visible au sein d'un ensemble lithique. Une partie de la production d'éclats ne se distingue pas de la production mettant en œuvre une percussion directe dure lancée dite «classique ». De plus, la similitude entre les caractéristiques décrites pour la percussion sur enclume et celles liées à la production et l'utilisation des pièces esquillées génère des doutes quant à l'interprétation de cette percussion comme technique de débitage stricto sensu.

\section{Percussion sur enclume et pièces esquillées}

Selon la définition proposée pour le Néolithique ancien, la pièce esquillée apparaît comme une pièce « ... présentant des enlèvements bifaciaux distaux et proximaux, plus rarement senestres et dextres, de morphologie en général écailleuse, et dont la face d'éclatement présente le plus souvent les ondes de fracture « vibrées » (Binder 1987). En ce sens, le problème de la différenciation entre les interprétations d'outil et de nucléus persiste. Ce problème n'est pas nouveau et les risques de confusion «nucléus/ outil » étaient déjà rapportés dès les premiers articles concernant la pièce esquillée (Bardon et al. 1906; Octobon 1938). Aujourd'hui, si elle n'est pas encore clairement démontrée d'un point de vue tracéologique (Astruc 1997 ; Gassin 1991 ; Beugnier 1997a et b), l'utilisation en tant que pièce intermédiaire de la pièce esquillée est globalement admise (Binder 1987 ; Gassin 1996). Selon cette hypothèse fonctionnelle, cet outil subit donc des chocs proximo-distaux propres à générer des stigmates proches de ceux résultant du débitage sur enclume (esquillements des parties proximales et distales, 
surface de fracture à ondes "vibrées», ...). La caractérisation pratique des deux hypothèses demeure donc un exercice délicat.

Cependant, l'étude de plusieurs séries lithiques campaniformes, et notamment celle du site du Mas de Vignoles IV à Nimes, Gard (Convertini et al. 2004; Furestier 2005) a permis de distinguer, directement ou indirectement, des pièces caractéristiques des deux cas :

- des éclats ne portant que très peu d'esquillements et/ou d'écrasements (et souvent majoritairement en partie proximale), à talons linéaires ou écrasés, et présentant quelquefois une finesse inapte à supporter un contrecoup violent sans risque de rupture ont été observés. Ils portent souvent des négatifs d'éclats réguliers sur leurs faces supérieures. Ces éclats sont le résultat d'une percussion sur enclume exercée sur des pièces interprétées comme des nucléus.

- inversement, des pièces robustes dont les parties proximales et distales (et aussi dextres et senestres) présentaient des retouches écailleuses «vibrées » en nombre (allant jusqu'à une morphologie très "écrasée ») et des étoilements témoignant de coups répétés ont été également observés. L'hypothèse de l'outil « pièce esquillée » a, dans ce cas, été privilégiée.

Ces deux observations attestent la présence de deux éléments distincts, pourtant typologiquement et technologiquement proches. Des pièces esquillées peuvent donc être des nucléus et/ou des outils. Toutefois, seules les pièces hautement caractéristiques peuvent être différenciées : les pièces esquillées ne présentant aucun négatif (laminaire ou d'éclat) pourront difficilement supporter le qualificatif de nucléus. À l'inverse, la présence de ces mêmes négatifs ne place pas de facto les pièces observées dans la classe des nucléus.

Les risques de recouvrement entre les critères de reconnaissance demeurent effectivement importants. Ces risques sont soulignés par des facteurs intrinsèques, et accentués par des limites interprétatives supplémentaires :

- l'intensité d'utilisation des pièces esquillées et la nature des matériaux travaillés peuvent générer des retouches différentes, plus ou moins étendues, et rendent la définition même de pièce esquillée incertaine ;

- de par sa nature et sa fonction supposée, la pièce esquillée est un outil dont la retouche, due à l'utilisation, masque la morphologie première du support de départ. Si un nucléus sur enclume est réutilisé comme pièce esquillée, la différenciation sera plus périlleuse. Plusieurs pièces à retouches envahissantes ne donnent donc pas d'information sur leur nature première ;

- inversement, selon la matière travaillée avec une pièce esquillée, son utilisation peut "produire » des éclats présentant les critères de reconnaissance des éclats débités sur enclume alors qu'ils ne sont pas des produits de première intention mais des déchets d'utilisation. Plusieurs éclats (ou fragments d'éclat) portant seulement une onde de fracture vibrée ne peuvent donc pas être départagés ;

Ces remarques soulignent les difficultés de caractérisation par la nature même de la pièce esquillée interprétée comme un outil ou un nucléus. De plus, d'autres possibilités d'interprétation des pièces esquillées et de la percussion sur enclume peuvent mettre en évidence de nouvelles limites, liées notamment à la réalisation d'un même geste technique. Ainsi, la majorité des stigmates observés peut être générée tant par l'utilisation des pièces esquillées que par la production d'éclats débités sur enclume. 
Outre la distinction outil/nucléus, il faut prendre également en compte celle entre outil et produit... Plusieurs pièces restent alors malaisées à définir (fig. $8 \mathrm{n}^{\circ} 6$ et 7).

31 L'hypothèse d'une percussion sur enclume mise en œuvre comme technique de façonnage exclue l'interprétation de nucléus et limite celle d'outil. Cependant, l'obtention d'un type de tranchant spécifique par façonnage sur enclume n'est pas confirmée par l'étude tracéologique (Gassin 1996). L'hypothèse du façonnage est alors uniquement proposée dans le cas d'un outil dont l'utilisation ne laisserai pas de traces ou bien dans le cas d'une phase de préforme d'un outillage encore indéterminé. Mais ici encore la rareté des études tracéologiques sur des pièces esquillées du Néolithique final ne permet pas de conclure...

32 L'ensemble des limites interprétatives des pièces esquillées et de la percussion sur enclume appelle nécessairement la multiplication des études traitant de ces deux sujets jusqu'alors délaissés par les recherches sur la fin du Néolithique. L'examen de nouvelles séries, mais aussi d'anciennes collections, doit permettre de confirmer ou d'infirmer les hypothèses exposées ici et de conforter ainsi le rôle de ces deux éléments dans la compréhension des cultures de la fin du troisième millénaire avant notre ère.

\section{Percussion directe dure et troisième millénaire}

Dans l'acception couramment entendue, la percussion directe dure ne peut être considérée comme un marqueur chronoculturel. Sa grande variabilité d'application au sein d'un même groupe culturel et son ubiquité sur l'ensemble du troisième millénaire, semblent interdire l'utilisation archéologique de cette technique comme élément datant. Cependant, l'étude approfondie de l'industrie lithique d'un contexte culturel précis dans une région donnée permet de nuancer ce constat a priori. Pour le Campaniforme du Sud-Est de la France, la percussion directe dure apparaît comme une technique expédiente. Mais cette définition doit ici s'entendre comme une optimisation des contraintes prises en compte dans la mise en œuvre de la production de l'outillage lithique et non dans le sens péjoratif souvent accolé au terme d'expédient. Focalisé sur la production d'un outillage plus restreint qu'auparavant, la percussion directe dure est la mieux adaptée aux changements de contraintes techniques et socio-économiques. La multiplication des groupes humains à la fin du Néolithique engendre un changement de statut social de l'industrie lithique qui voit le développement et la diffusion des productions spécialisées à haute valeur de signe (Renault 1998; Briois 2005 ; Mallet et al. à paraître), ainsi qu'un changement dans l'approvisionnement en matières premières lié à une nouvelle gestion des territoires et des ressources. En tant que nouveau groupe culturel dans ce contexte, le Campaniforme illustre bien l'adaptation à ces nouvelles contraintes. Combinant l'exploitation d'un territoire local restreint et la variabilité qualitative des matières premières qu'ils y prélèvent, les Campaniformes ont mis en oeuvre la percussion directe dure comme moyen principal d'adaptation à ces contraintes et de réponse à leurs besoins. Ce qui peut apparaître comme un pis-aller technique doit au contraire être vu comme un réel choix culturel. L'ensemble de l'industrie lithique campaniforme montre en effet que ce choix technique ne saurait être considéré comme une carence de savoir faire des tailleurs: certaines pièces témoignent d'un important investissement technique et l'analyse des ressources disponibles montre que la production d'un module d'éclat préétabli à partir de matières premières de qualité très variable ne doit rien aux contraintes précitées puisque 
certains territoires exploités présentent des gîtes de matières de bonne qualité qui n'ont pas été choisis.

La percussion directe dure et les diverses modalités d'application exposées ici confirment cette hypothèse de choix culturel caractérisé par les notions d'adaptation et d'optimisation. Au même titre que les productions expédientes des périodes précédentes (Léa 2005), celles des Campaniformes s'imposent comme des révélateurs du statut social des industries lithiques et des sites.

\section{BIBLIOGRAPHIE}

ALLARD P. 2005 - Interrogations sur la notion des productions dites « expédientes » dans l'industrie lithique danubienne du quart nord-occidental de l'Europe. In : ASTRUC L. (Dir.), Table ronde «Au-delà de la notion de technologie expédiente » : outillages lithiques et osseux au Néolithique, Nanterre, Umr ArScAn, p. 180-185 (Cahier des thèmes transversaux ArScAn V 2003-2004).

ASTRUC L. 1997 - Analyse tracéologique de quelques artéfacts en silex. In : OTHENIN-GIRARD B. (Dir.) Le Campaniforme d'Alle, Noir Bois (Jura, Suisse), Porrentruy, Société jurassienne d'Emulation, p. 121-127 (Cahiers d'Archéologie jurassienne, 7)

ASTRUC L. (dir.) 2005 - Au-delà de la notion de technologie expédiente, Table ronde du 15 mars 2004, Cahiers des thèmes transversaux ArScAn, Tome V (2003-2004), Thème 3 (« Systèmes de production et de circulation »), Nanterre, Umr ArScAn, p. 174-227.

ASTRUC L., BON F., LEA V., MILCENT P.-Y., PHILIBERT S., (dir.) à paraître - Normes techniques et pratiques sociales : de la simplicité des outillages pré- et protohistoriques. XXIIIe Rencontres Internationales d'Archéologie et d'Histoire d'Antibes, Antibes : Editions APDCA, à paraître.

BARDON L. et BOUYSSONIE A. et J. 1906 - Outils écaillées par percussion à la Coumba del Bouitou (Corrèze), Revue de l'Ecole d'Anthropologie, tome XVI, pp. 170-175, 5 fig.

BEUGNIER V. 1997a - Analyse tracéologique de l'industrie en silex du niveau VIII. In PETREQUIN P. éd. Les sites littoraux néolithiques de Clairvaux et Châlain (Jura) III Châtain. Station III, 3200-2900 av. J.-C. Paris ; Éditions de la Maison des sciences de l'homme.

BEUGNIER V. 1997b - L'usage du silex dans l'acquisition et le traitement des matières animales dans le Néolithique de Chalain et Clairvaux. La-Motte-aux-Magnins et Châtain 3 (Jura, France). Nanterre, Université de Paris X, 492 p. (thèse de Doctorat de l'Université).

BINDER D. 1987 - Le Néolithique ancien provençal. Typologie et technologie des outillages lithiques, Paris : Editions du CNRS, 1987, 209 p. (XXIVe supplément à Gallia Préhistoire).

BRIOIS F. 2005 - Les industries de pierre taillée néolithiques en Languedoc occidental. Nature et évolution des outillages entre les 6ème et 3ème millénaires av. J.C. Lattes : Publications de l'UMR 154 du CNRS, 2005, 341 p. (Monographies d'Archéologie Méditerranéenne, n²0).

CONVERTINI F. 1996 - Production et signification de la céramique campaniforme à la fin du 3ème millénaire av. J.C. dans le Sud et le Centre-Ouest de la France et en Suisse occidentale, Oxford : Tempus Reparatum, 1996, 372 p., 111 fig., 71 tabl., 2 pl. (B.A.R., International Series 656). 
CONVERTINI F., FURESTIER R., ASTRUC L., FOREST V. et JALLOT L. 2004 - Le mas de Vignole IV à Nîmes (Gard) : Résultas préliminaires des fouilles d'un fossé à occupation campaniforme. In : Dartevelle H. (dir.) Auvergne et Midi : 5ème Rencontres Méridionales de Préhistoire Récente, Session de Clermont-Ferrand, octobre 2002, pp 493-507.

DETREY J. 2002 - Mise en évidence d'une composante régionale dans l'industrie lithique campaniforme d'Ajoie (Jura, Suisse). In : M. BAILLY, T. PERRIN, R. FURESTIER (dir.) Les industries lithiques taillées en Bassin rhodanien : problèmes et actualités. Montagnac, éditions M. Mergoil, p. 181-204. (Préhistoires, 8).

FURESTIER R. 2005 - Les industries lithiques campaniformes du sud-est de la France, Thèse de l'Université de Provence, Aix-en-Provence, 2005, 3 vol. , 738 p.

FURESTIER R. 2007 - Les industries lithiques campaniformes du sud-est de la France. Oxford, 2007, 339 p. (BAR International Series 1684).

GASSIN B. 1991 - Étude fonctionnelle. in D. Binder, Une économie de chasse au Néolithique Ancien. La grotte Lombard à Saint-Vallier-de-Thiey (Alpes-Maritimes/. Paris : CNRS : 51-60. (Monographies du Centre de recherches archéologiques, 5).

GASSIN B. 1996 - Evolution socio-économique dans le Chasséen de la grotte de l'Eglise supérieure (Var). Apport de l'analyse fonctionnelle des industries lithiques. Paris, C.N.R.S. éditions, 326 p. (Monographies du CRA. $n^{\circ} 17$ ).

GASSIN B. et BINDER D. Avec la coll. de SENEPART I. 2004 - Statut et fonction des productions d'éclats au Néolithique : exemples provençaux. In : Congrès Préhistorique de France, XXV e session, Nanterre 24-26 novembre 2000, Approches fonctionnelles en Préhistoire, Mémoires de la S.P.F. Paris, p. 167-179.

GUYODO J.N. et MARCHAND G. 2005 - La percussion bipolaire sur enclume dans l'ouest de la France de la fin du Paléolithique au Chalcolithique : une lecture économique et sociale, Bulletin de la Société Préhistorique Française, tome 102, n³ 2005, p. 539-550.

JOUSSAUME R. 1981 - Le Néolithique de l'Aunis et du Poitou occidental dans son cadre atlantique, Rennes, Université, 625 p. (Travaux du Laboratoire d'Anthropologie, Préhistoire, Protohistoire et Quaternaire armoricains)

LEA V. 2005 - Qui peut le plus peut le moins. In : Au-delà de la notion de technologie expédiente, Table ronde organisée par Laurence Astruc, 15 mars 2004, Cahiers des thèmes transversaux ArScAn, Tome V (2003-2004), Thème 3 ("Systèmes de production et de circulation »), Paris, p. 186-192.

LECH J. 1982/1983 - Flint work of the early farmers. Production trends in central european chipping industry from 4500-1200 B.C and outline. Acta Archeologica carpatica Tome XXII 1982/83 p. 5-64.

LEMERCIER O. 2004 - Les Campaniformes dans le sud-est de la France, Lattes : Publications de l'UMR 154 du CNRS, 2004, 515 p. (Monographies d'Archéologie Méditerranéenne, nº 18).

MALLET N., IHUEL E. et VERJUX C. à paraître - La diffusion du silex du Grand-Pressigny au sein des groupes des IVème et IIIème millénaires. In : DIAS-MERHINO M.H., LEA V., BAILLY M., BRIOIS F., FOUERE P., GERNIGON K. et VALDEYRON N. (dir.) Les industries lithiques taillées des IVème et IIIème millénaires en Europe occidentale, 7-9 avril 2005, Toulouse, à paraître.

OCTOBON E. 1938 - Contribution à l'étude des outillages, ciseaux et pièces esquillées, B.S.P.F., tome 35, pp. 409-412. 
PELEGRIN J. 2000 - Les techniques de débitage au Tardiglaciaire : critères de diagnose et quelques réflexions. In : B. VALENTIN, P. BODU et M. CHRISTENSEN (éd.) L'Europe centrale et Septentrionale au Tardiglaciaire. Confrontation des modèles régionaux de peuplement. Actes de la Table-ronde internationale de Nemours, mai 1997. Nemours, éd. A.P.R.A.I.F., p. 73-86 (mémoires du Musée de Préhistoire d'Ile-de-France, 7).

PERLES C. 1991 - Economie des matières premières et économie du débitage, deux conceptions opposées ? In : 25 /vingt cinq/ ans d'études technologiques en préhistoire. Bilan et perspectives : Actes des 11e rencontres internationales d'archéologie et d'histoire d'Antibes, 18-20 Octobre 1990, APDCA, CNRS (Dir.), Juan-les-Pins, APDCA, p. 35-45 (Rencontres internationales d'Archéologie et d'Histoire d'Antibes, 11)

RENAULT S. 1998 - Economie de la matière première. L'exemple de la production, au Néolithique final, en Provence, des grandes lames en silex zoné oligocène du bassin de Forcalquier (Alpes-deHaute-Provence. In : D'ANNA A., BINDER D. (Dir.) : Production et Identité culturelle, Actualités de la Recherche, Actes des Rencontres Méridionales de Préhistoire Récente, deuxième session, Arles, novembre 1996, Antibes : Editions APDCA, 1998, p. 145-161.

\section{NOTES}

1. Ce mode peut être techniquement comparé à la fracture dite en "split ", voire même au «débitage côtier» (Joussaume 1981). Cependant, ces dénominations sont archéologiquement connotées et n'ont pas été retenues afin d'éviter les risques de confusion supplémentaires.

2. Comme pour l'ensemble de cette étude, tous les stigmates décrits sont observés uniquement sur des éléments en silex, les autres matières premières n'intervenant que sporadiquement dans les assemblages campaniformes (Furestier 2005).

\section{RÉSUMÉS}

Au-delà des qualificatifs dépréciatifs concernant l'ubiquité et la simplicité de la percussion directe à la pierre dure, son étude technologique en contexte chronoculturel campaniforme a permis de mettre en évidence la variabilité de ses applications à la fin du troisième millénaire.

Ainsi, en plus d'une caractérisation de la percussion directe classique, une percussion sur enclume a été observée. Malgré les risques de recouvrements entre les critères de reconnaissance de ces deux techniques, quelques spécificités telles que la morphologie des nucléus et les caractères principaux des éclats produits ont été définis. L'analyse des nucléus et des produits permet également la distinction de deux modalités d'application de la percussion sur enclume. L'aspect général de simplicité lié à l'approvisionnement local en matière première, l'inorganisation apparente du débitage et l'exploitation opportuniste des nucléus s'opposent au constat de diversité de mise en œuvre de la technique la plus répandue de la Préhistoire. En tant qu'adaptation à l'ensemble des contraintes socio-économiques, la percussion directe dure et sa variante sur enclume sont alors interprétées selon l'idée d'optimisation et de réel choix culturel, et non plus selon celle de dégénérescence technique.

Cependant, du point de vue de l'étude technologique des industries lithiques campaniformes, la 
caractérisation de ces techniques et de leur variabilité rappelle les difficultés de distinction de plusieurs types de pièces entre elles. Le cas de la pièce esquillée et des problèmes d'interprétation qu'elle entraîne illustre bien ces difficultés. Toutefois, la multiplication des études des industries lithiques $\mathrm{du}$ troisième millénaire avant notre ère fait progressivement reculer les limites interprétatives. La percussion sur enclume, et plus largement l'ensemble des productions dites expédientes, apparaissent alors comme des éléments importants de définition des statuts sociaux et culturels des sociétés de la fin de la Préhistoire.

Beyond the depreciative commentaries concerning the ubiquity and the simplicity of hard-stone direct percussion techniques, the technological study within its bell-beaker chronocultural context, has enabled us to better clarify the variability of its applications at the end of the third millenium.

In addition to a characterisation of the classical direct percussion, anvil percussion has also been observed. In spite of the difficulty to distinguish the traces of the two techniques, certain characteristics such as the morphology of the core and the principal facets of the flakes produced have been defined. The analysis of the cores and its products also allow us to show two techniques of anvil percussion. The general impression of simplicity arising from the local provenance of the raw material, the apparent non-ordered character of the debitage and opportunistic exploitation of the cores are opposed to the observations of the great diversity of practice known in the prehistoric period. As an adaptation to the prevailing socio-economic constraints, hard direct percussion and its anvil variant are thus interpreted according to an idea of optimisation and of real cultural choice and no longer a simple technical decadence.

However, from the point of view of the techological study of bell beaker lithic industries, the characterisation of these techniques and their variability bring us back to the difficulty to distinguish between certain kinds of object. This difficulty may be illustrated by the case of splintered pieces and the interpretation difficulties arising. Neverthless, the multiplication of the studies treating lithic industries of the third millenium BC is gradually pushing back these interpretative limits. Anvil percussion, and on a wider scale, the whole suite of production techniques termed "expedient", begin to emerge as social and cultural status significant markers for the societies at the end of the pre-historical period.

\section{INDEX}

Mots-clés : néolithique final, Campaniforme, sud-est de la France, percussion directe dure, percussion sur enclume, pièces esquillées

Keywords : Later Neolithic, Bell Beaker culture, south-east of France, hard direct percussion, hard direct percussion on anvil, splintered pieces

\section{AUTEUR}

\section{ROBIN FURESTIER}

UMR 5140 Archéologies des Sociétés Méditerranéennes 